\title{
The Equivalent Thermal Conductivity of the Micro/Nano Scaled Periodic Cubic Frame Silver and Its Thermal Radiation Mechanism Analysis
}

\author{
Haiyan Yu, Haochun Zhang*D, Heming Wang and Dong Zhang
}

Citation: Yu, H.; Zhang, H.; Wang,

H.; Zhang, D. The Equivalent

Thermal Conductivity of the

Micro/Nano Scaled Periodic Cubic

Frame Silver and Its Thermal

Radiation Mechanism Analysis.

Energies 2021, 14, 4158.

https://doi.org/10.3390/en14144158

Academic Editor: Ron Zevenhoven

Received: 19 May 2021

Accepted: 5 July 2021

Published: 9 July 2021

Publisher's Note: MDPI stays neutral with regard to jurisdictional claims in published maps and institutional affiliations.

Copyright: (C) 2021 by the authors. Licensee MDPI, Basel, Switzerland. This article is an open access article distributed under the terms and conditions of the Creative Commons Attribution (CC BY) license (https:/ / creativecommons.org/licenses/by/ $4.0 /)$.
School of Energy Science and Engineering, Harbin Institute of Technology, Harbin 150001, China; yuhaiyan22@foxmail.com (H.Y.); 20S102105@stu.hit.edu.cn (H.W.); 20S002016@stu.hit.edu.cn (D.Z.)

* Correspondence: hczhang@hit.edu.cn

\begin{abstract}
Currently, there are few studies on the influence of microscale thermal radiation on the equivalent thermal conductivity of microscale porous metal. Therefore, this paper calculated the equivalent thermal conductivity of high-porosity periodic cubic silver frame structures with cell size from $100 \mathrm{~nm}$ to $100 \mu \mathrm{m}$ by using the microscale radiation method. Then, the media radiation characteristics, absorptivity, reflectivity and transmissivity were discussed to explain the phenomenon of the radiative thermal conductivity changes. Furthermore, combined with spectral radiation properties at the different cross-sections and wavelength, the radiative transmission mechanism inside high-porosity periodic cubic frame silver structures was obtained. The results showed that the smaller the cell size, the greater radiative contribution in total equivalent thermal conductivity. Periodic cubic silver frames fluctuate more in the visible band and have better thermal radiation modulation properties in the near infrared band, which is formed by the Surface Plasmon Polariton and Magnetic Polaritons resonance jointly. This work provides design guidance for the application of this kind of periodic microporous metal in the field of thermal utilization and management.
\end{abstract}

Keywords: micro scale; thermal radiation; equivalent thermal conductivity; thermal radiation mechanism

\section{Introduction}

Since the discovery of microscale thermal radiation effects by Tian et al. [1,2] in the 1960s, these phenomena have attracted scholars' attention worldwide. Particularly in recent years, with the increasing maturity of the microscale processing and measurement technology, whose thermal radiation properties and modulation methods in the direction of solar radiation, photoelectric conversion, infrared detection, optical imaging, infrared stealth, etc. have become a research hotspot with a highly practical application value. Within the microscale, the heat transfer between two or more close objects can far exceed blackbody radiation, where the Planck blackbody radiation law cannot function anymore. Therefore, the explanation of the heat transfer phenomenon of macroscopic objects is no longer applicable for the object whose characteristic size is comparable to or smaller than the wavelength of thermal radiation [3]. In comparison to macroscopic radiation, the phenomena like Surface Plasmon Polaritons (SPPs) [4-6], Phonon Polariton Effect [7,8], Photonic Band-gap Effect [9], Photon Tunneling Effect [10], etc. produced in the field of microscale thermal radiation.

Various scholars have focused on the research of the thermal radiation properties of periodic microstructures. Lee et al. [11,12] calculated the emissivity of silver structures using rigorous coupled-wave analysis and found that the emissivity can reach large emission peaks at resonant frequencies with excited magnetic polarization. Wang et al. [13] investigated the effect of complex grating structures on thermal radiation properties using a timedomain finite-difference method. Qiu et al. [14] researched thermal radiation properties of random rough surfaces. Huang et al. [15,16] analyzed the thermal radiation properties 
of circular microcavity arrays and grating-coupled thermochromic materials, as well as the infrared negative refractive index properties of microstructures. Xuan's group $[17,18]$ discussed the effect of near-field thermal radiation on graphene, and graphene's thermal control properties with different material surfaces covered. Liu et al. $[19,20]$ analyzed the spectral radiation characteristics of the microscale rough ligament surface inside the open-cell nickel foam.

However, scholars pay more attention on the study of surface or media thermal radiative properties, instead of the equivalent thermal conductivity of microscale porous metal. Therefore, in this paper, the equivalent thermal conductivity of periodic cubic frame silver structures was calculated by the finite element method, where porosities are 0.896 , $0.784,0.684$ and 0.500 , respectively and cell size between $100 \mathrm{~nm}$ and $100 \mu \mathrm{m}$. In order to explain the effect of microscale effects on the equivalent radiative thermal conductivity, the further work analyzed the absorption, reflection and transmission capabilities of each model. Moreover, the electric field direction and magnetic field intensity distribution at the appearance of absorption and reflection peaks in the near-infrared band are analyzed, thereby giving a reasonable explanation for the thermal radiation properties of periodic cubic frame silver microstructures.

\section{Mathematical Methods}

Finite element method (FEM) [21-23] as a highly adaptable numerical method used wildly in the calculation of thermal radiation properties of microstructures. By dividing a continuous three-dimensional region into a finite number of small regions, FEM applied to any physical field described by differential equations theoretically, based on the variational principle. The following work was carried out by using FEM.

\subsection{Absorptivity, Reflectivity and Transmissivity}

The research of microscale thermal radiation is generally presented in terms of electromagnetic theory [24], with describing the distribution of electromagnetic field by Maxwell equations in vacuum as [24]:

$$
\left\{\begin{array}{c}
\nabla \times \boldsymbol{H}=\frac{\partial \boldsymbol{D}}{\partial t}+\boldsymbol{J} \\
\nabla \times \boldsymbol{E}=-\frac{\partial \boldsymbol{B}}{\partial t} \\
\nabla \cdot \boldsymbol{B}=0 \\
\nabla \cdot \boldsymbol{D}_{e}=\rho
\end{array}\right.
$$

where $B$ indicates the magnetic flux density, $\mathrm{Wb} / \mathrm{m}^{2} ; \boldsymbol{D}$ stands for the electric flux density while $D_{e}$ is the electric displacement vector, $\mathrm{C} / \mathrm{m}^{2} ; E$ presents the electric field, $\mathrm{V} / \mathrm{m}$; $H$ is the magnetic field, A/m; $J$ indicates the current density, A $/ \mathrm{m}^{2} ; t$ is the time, s, $\rho$ is the volume charge density, $\mathrm{C} / \mathrm{m}^{3}$. To obtain the Maxwell equations in media instead of vacuum, the material equations are needed as [24]:

$$
\left\{\begin{array}{c}
D_{e}=\varepsilon \boldsymbol{E} \\
\boldsymbol{B}=\mu \boldsymbol{H} \\
\boldsymbol{J}=\sigma \boldsymbol{E}
\end{array}\right.
$$

where $\varepsilon$ indicates the permittivity, $\mathrm{F} / \mathrm{m} ; \mu$ is permeability, $\mathrm{H} / \mathrm{m} ; \sigma$ indicates the conductivity, $\mathrm{S} / \mathrm{m}$. Bring Equation (2) into Equation (1), the obtained Maxwell equations in media as Equation (3) [24]:

$$
\left\{\begin{array}{c}
\nabla \times \boldsymbol{H}=\varepsilon \frac{\partial \boldsymbol{E}}{\partial t}+\sigma \boldsymbol{E} \\
\nabla \times \boldsymbol{E}=-\mu \frac{\partial \boldsymbol{H}}{\partial t} \\
\nabla \cdot \boldsymbol{H}=0 \\
\nabla \cdot \boldsymbol{E}=\frac{\rho}{\varepsilon}
\end{array}\right.
$$

By considering only a cubic microstructure, the absorption capacity of an object can be expressed in terms of the ratio of the rate of absorbed energy to the incident energy, which 
is the absorption cross section. In electromagnetic theory, the absorption cross section $Q^{(a)}$ is indicated as [25]:

$$
Q^{(\mathrm{a})}=\frac{W^{(\mathrm{a})}}{\left|\left\langle\boldsymbol{S}^{(\mathrm{i})}\right\rangle\right|}=\frac{-\operatorname{Re} \iint_{\Sigma}\left\{\boldsymbol{E}^{(\mathrm{i})} \times \boldsymbol{H}^{(\mathrm{s}) *}+\boldsymbol{E}^{(\mathrm{s})} \times\left(\boldsymbol{H}^{(\mathrm{i}) *}-\boldsymbol{H}^{(\mathrm{s}) *}\right)\right\} \cdot \mathbf{n} d A}{\operatorname{Re}\left\{\boldsymbol{E}^{(\mathrm{i})} \times \boldsymbol{H}^{(\mathrm{i}) *}\right\}}
$$

where $\boldsymbol{E}^{(\mathrm{s})}$ and $\boldsymbol{H}^{(\mathrm{s})}$ indicate the scattered electric and magnetic fields, respectively, $\boldsymbol{E}^{(\mathrm{i})}$ and $\boldsymbol{H}^{(\mathrm{i})}$ the incident electric and magnetic fields, respectively, ${ }^{*}$ indicates the conjugate complex vector, $W^{(a)}$ indicates the absorption rate, $\langle\cdot\rangle$ represents the system average, and $S^{(i)}$ indicates the incident energy flow, also known as the Poyntingvector. When the microelements form a periodic monolayer structure, the absorption capacity can be represented by the absorption rate [25]:

$$
\alpha_{\mathrm{A}}=1-\frac{\int\left(\boldsymbol{S}^{(\mathrm{r})}+\boldsymbol{S}^{(\mathrm{t})}\right) \cdot \mathbf{n d} A}{\int \boldsymbol{S}^{(\mathrm{i})} \cdot \mathbf{n d} A}
$$

where $S^{(\mathrm{r})}$ indicates the reflected energy flow and $S^{(\mathrm{t})}$ indicates the transmitted energy flow. $\alpha_{\mathrm{A}}$ indicates the absorption rate. Correspondingly, the reflectivity $\alpha_{\mathrm{R}}$ and transmissivity $\alpha_{\mathrm{T}}$ are represented in Equation (6) as follows [25]:

$$
\alpha_{\mathrm{R}}=\frac{\int \boldsymbol{S}^{(\mathrm{r})} \cdot \mathbf{n d} A}{\int \boldsymbol{S}^{(\mathrm{i})} \cdot \mathbf{n d} A} \text { and } \alpha_{\mathrm{T}}=\frac{\int \boldsymbol{S}^{(\mathrm{t})} \cdot \mathbf{n d} A}{\int \boldsymbol{S}^{(\mathrm{i})} \cdot \mathbf{n d} A}
$$

In this paper, the Equations (5) and (6) would use to calculate the absorption, reflection and transmittance of periodic cubic silver structures.

In this research, the transfer matrix method (TMM) is used to verify the correctness of the FEM calculation in Section 3.1, which is almost exact analytical solution. The media numbers on both sides of the Air-Silver-Air structure are 0 to $I+1$. When the wavelength $\lambda$ incident on the multilayer films, the transmission matrix of the medium as follows [26]:

$$
M_{i}=\left[\begin{array}{cc}
\cos \zeta_{i} & \frac{j}{n_{i}} \sin \zeta_{i} \\
j n_{i} \sin \zeta_{i} & \cos \zeta_{i}
\end{array}\right]
$$

where $\zeta_{i}=k n_{\mathrm{i}} d_{\mathrm{i}}$, is the effective phase shift thickness of the film layer, $d_{\mathrm{i}}$ is the thickness of this layer, $n$ stand its refractive index, and the wave vector $k=2 \pi / \lambda$. The transfer matrix of the multilayer is [26]:

$$
M=\prod_{i=1}^{t} M_{i}=\left(\begin{array}{ll}
m_{11} & m_{12} \\
m_{21} & m_{22}
\end{array}\right)
$$

Therefore, the electric and magnetic fields on both sides of the multilayer membrane structure can be calculated in Equation (9) [26]:

$$
\left[\begin{array}{l}
E_{0} \\
H_{0}
\end{array}\right]=M_{1} \cdot M_{2} \cdot M_{3} \cdots M_{t}=\left(\begin{array}{cc}
m_{11} & m_{12} \\
m_{21} & m_{22}
\end{array}\right)\left[\begin{array}{c}
E_{t+1} \\
H_{t+1}
\end{array}\right]
$$

where $E_{0}, H_{0}, E_{i+1}$, and $H_{i+1}$, respectively represent electromagnetic fields at the outer surface of the multilayer films. When the electromagnetic wave is incident vertically, the reflection coefficient $r$ and transmission coefficient $t$ of the multilayer film structure are [26]:

$$
\begin{aligned}
& \boldsymbol{r}=\frac{\left(m_{11}+m_{12} n_{t+1}\right) n_{0}-\left(m_{21}+m_{22} n_{t+1}\right)}{\left(m_{11}+m_{12} n_{t+1}\right) n_{0}+\left(m_{21}+m_{22} n_{t+1}\right)} \\
& \boldsymbol{t}=\frac{2 n_{0}}{\left(m_{11}+m_{12} n_{t+1}\right) n_{0}+\left(m_{21}+m_{22} n_{t+1}\right)}
\end{aligned}
$$


The $\alpha_{A}, \alpha_{R}$ and $\alpha_{T}$ can be further obtained from the reflectance and transmittance as follows [26]:

$$
\alpha_{R}=|\boldsymbol{r}|^{2}, \alpha_{T}=\frac{n_{t+1}}{n_{0}}|\boldsymbol{t}|^{2} \text { and } \alpha_{A}=1-\alpha_{R}-\alpha_{T}
$$

\subsection{The Equivalent Thermal Conductivity}

Based on Equation (4), the spectral absorption coefficient $\beta_{\alpha}$ and a spectral scattering coefficient $\beta_{s}$ can be calculated as follows [27]:

$$
\begin{gathered}
\beta_{a}=\frac{-\operatorname{Re} \iint_{\Sigma}\left\{\boldsymbol{E}^{(\mathrm{i})} \times \boldsymbol{H}^{(\mathrm{s}) *}+\boldsymbol{E}^{(\mathrm{s})} \times\left(\boldsymbol{H}^{(\mathrm{i}) *}-\boldsymbol{H}^{(\mathrm{s}) *}\right)\right\} \cdot \mathbf{n} d A}{N \cdot \operatorname{Re}\left\{\boldsymbol{E}^{(\mathrm{i})} \times \boldsymbol{H}^{(\mathrm{i}) *}\right\}} \\
\beta_{s}=\frac{\operatorname{Re} \iint_{\Sigma}\left\{\boldsymbol{E}^{(\mathrm{s})} \times \boldsymbol{H}^{(\mathrm{s}) *}\right\} \cdot \mathbf{n} d A}{N \cdot \operatorname{Re}\left\{\boldsymbol{E}^{(\mathrm{i})} \times \boldsymbol{H}^{(\mathrm{i}) *}\right\}}
\end{gathered}
$$

where $A$ is the unit cross-sectional area and $N$ is the number of particles per unit volume.

The spectral extinction coefficient $\beta_{e}$ can be calculated as follows [27]:

$$
\beta_{e}=\beta_{a}+\beta_{s}
$$

According to the optical thickness approximation, the equivalent radiative thermal transfer equation is as follows [27]:

$$
\kappa_{\mathrm{rad}}=\frac{16 \sigma_{\mathrm{SB}} T_{\mathrm{m}}^{3}}{3 \beta_{e, \mathrm{R}}}
$$

where $\sigma_{\mathrm{SB}}$ is the Stefan-Boltzmann constant, $\kappa_{\text {rad }}$ is the radiative equivalent thermal conductivity and $\beta_{e, \mathrm{R}}$ is the Rosseland average extinction coefficient, which can be calculated as [24]:

$$
\frac{1}{\beta_{e, \mathrm{R}}}=\frac{\int_{0}^{\infty} \frac{1}{\beta_{e}} f(\lambda, T) d \lambda}{\int_{0}^{\infty} f(\lambda, T) d \lambda}
$$

where $\lambda$ is the wavelength, $f(\lambda, T)$ is the spectral distribution of Planck blackbody emission, given by [24]:

$$
f(\lambda, T)=\frac{\partial e_{\mathrm{b}, \lambda}}{\partial T}=\frac{C_{1}}{\lambda^{5}\left(e^{C_{2} / \lambda T}-1\right)}
$$

where $e_{\mathrm{b}, \lambda}$ is the blackbody spectral intensity, $C_{1}=2 \pi h c^{2}$ is the first radiation constant, $C_{2}=h c / k_{\mathrm{B}}$ is the second radiation constant and $h$ is the Planck constant [28]. Therefore, Equation (16) would be used to calculate the $\kappa_{\text {rad }}$ in the following calculation.

According to Fourier's law, the governing equations of the thermal conduction progress of a three-dimensional object can be established during the transient temperature field $T(x, y, z, t)$ as $[27,29]$ :

$$
\rho c \frac{\partial T}{\partial t}-\frac{\partial}{\partial x}\left(\kappa_{x} \frac{\partial T}{\partial x}\right)-\frac{\partial}{\partial y}\left(\kappa_{y} \frac{\partial T}{\partial y}\right)-\frac{\partial}{\partial z}\left(\kappa_{z} \frac{\partial T}{\partial z}\right)=q A^{\prime}
$$

where $\kappa_{x}, \kappa_{y}$, and $\kappa_{z}$ are the thermal conductivities of the object along the $x, y$ and $z$ directions, all three values of which are equal to $\kappa_{S}$ for isotropic materials, $A^{\prime}$ is the area. In this research, the thermal conductivities of pure Ag at room temperature $(T=300 \mathrm{~K})$ were adopted from Young's [30] experimental measurements, that is $\kappa_{s}=406 \mathrm{~W} /(\mathrm{m} \cdot \mathrm{K}) ; \rho$ is the density of the object $\mathrm{kg} / \mathrm{m}^{3}$; $c$ is the specific heat capacity of the object $J /(\mathrm{kg} \cdot \mathrm{K})$ and $q$ is the 
density of the thermal source in the object, $\mathrm{W} / \mathrm{m}^{2}$. According to Fourier's law, for the heat flux $q_{\mathrm{n}}$ through differential element, the Equation (20) is obtained [29]:

$$
q_{\mathrm{n}}=-\kappa_{\text {cond }} \frac{\partial T}{\partial n}
$$

where $\kappa_{\text {cond }}$ is the equivalent conductive thermal conductivity, $n$ presents the heat transfer direction. It should note that, the thermal conduction progress without consider radiation. The total equivalent thermal conductivity $\kappa_{\text {total }}$ is obtained by [28]:

$$
\kappa_{\text {total }}=\kappa_{\text {cond }}+\kappa_{\text {rad }}
$$

Therefore, the Equation (21) would be used to calculate the $\kappa_{\text {total }}$ in the following calculation.

\section{Physical Model}

In this paper, the three-dimensional geometric model established with simplified cubic hollow as shown in Figure 1. Silver was selected as the material of the infinite plate and periodic cubic structure for its good physical properties, with regardless of the oxidation condition and the effect of the changes in the $n, k$ value of the material. The cubic skeleton material chosen is metallic silver which is extensively used in photonic crystals. In consideration of the effect of wavelength on $n, k$, this curve was obtained by interpolating the experimental points measured by Yang et al. [31] using Bessel spline function as shown in Figure 2.

(a)

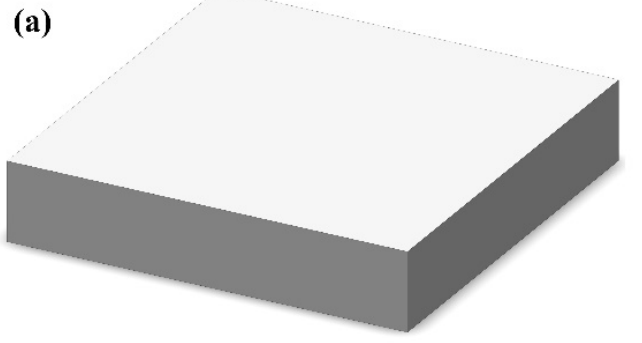

(b)

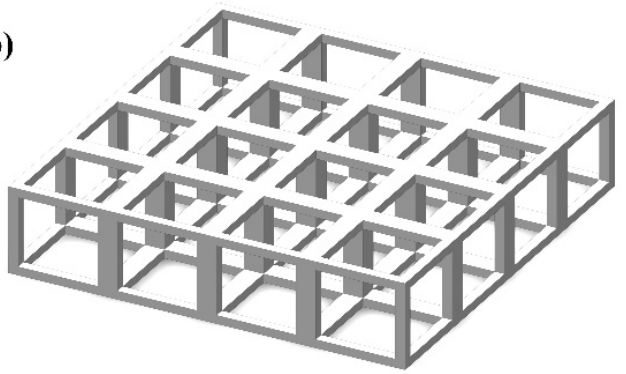

Figure 1. (a) Silver plate; (b) periodic cubic frame silver structure.
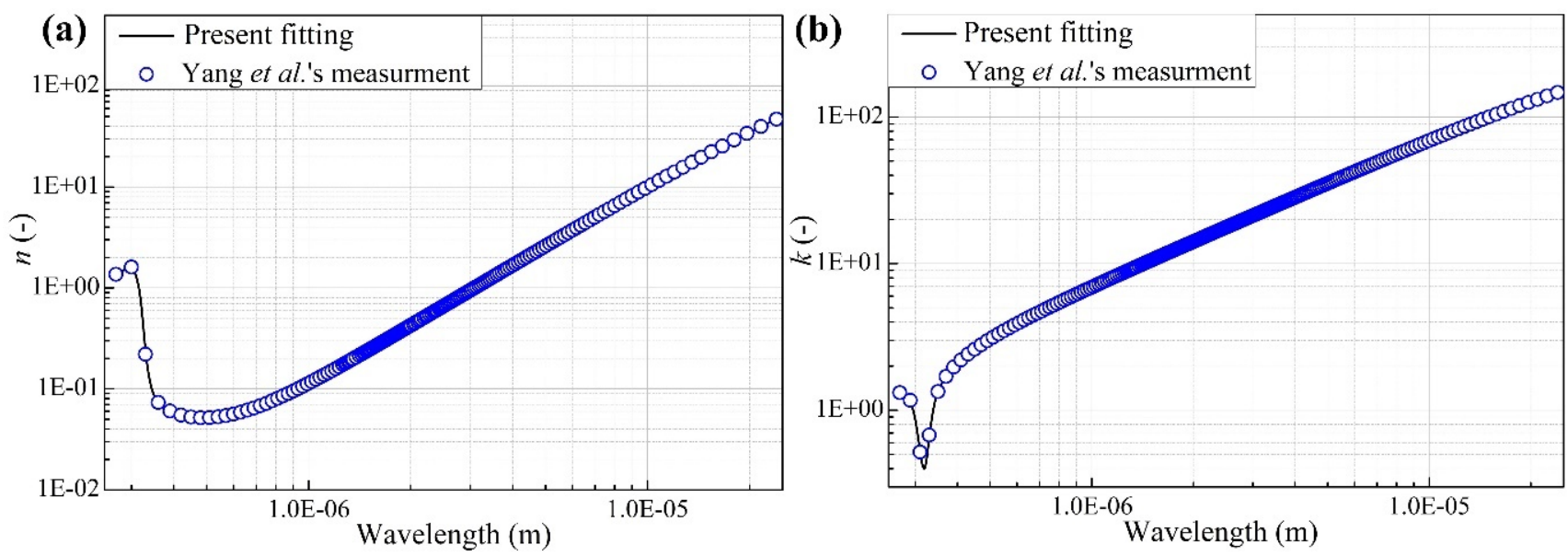

Figure 2. The optical constants of silver versus wavelength: (a) refractive index; (b) extinction index. 


\subsection{Calculation Domain of Infinity Silver Plate}

In order to verify the numerical simulation method, the infinite silver plate with thickness of $500 \mathrm{~nm}$ is calculated using the transmission matrix and finite element method, respectively, and the absorption curves are plotted using the obtained results as shown in Figure 3. In this section, Transverse Electromagnetic Wave (TEW) is used for vertical incidence. Due to the excitation effect of metals at far-infrared wavelengths at the microscale, it is impossible to obtain a more regular modulated wavelength range, therefore the wavelength range is taken as visible and mid-infrared wavelengths, which are $300 \mathrm{~nm}-3 \mu \mathrm{m}$.

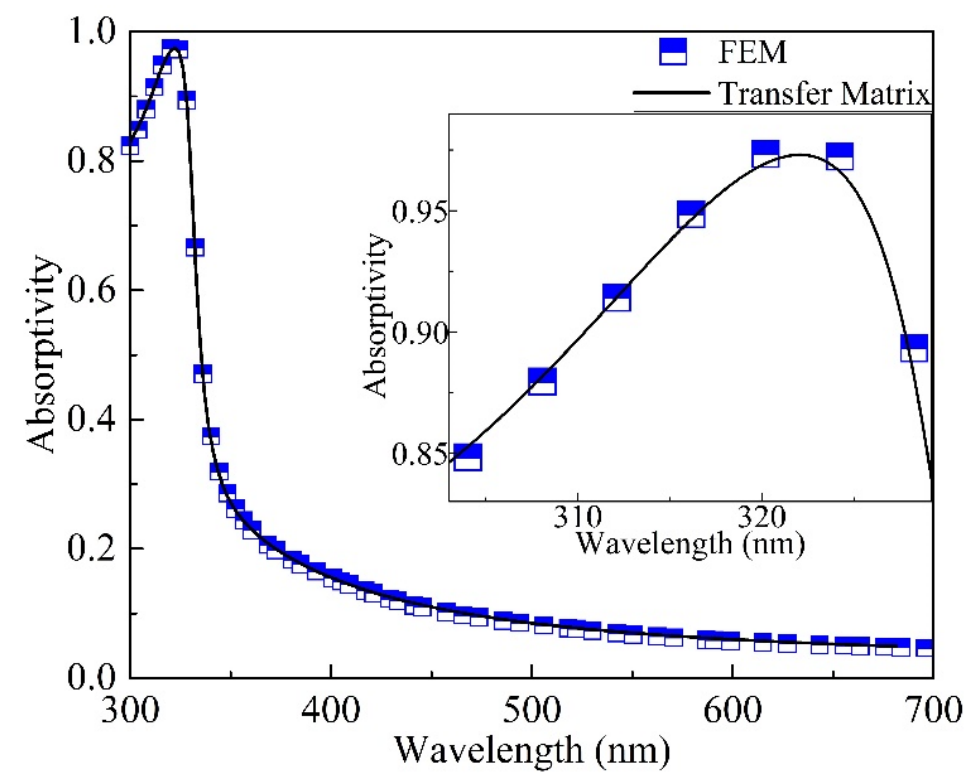

Figure 3. Absorptivity of infinite silver plate calculated by FEM and TMM.

As shown in Figure 3, the calculated results using FEM almost overlap with the absorptance curve obtained by the Transfer Matrix method (TMM), which is almost an exact analytical solution. The absorptivity increases with wavelength and then decreases sharply, with a maximum peak at $0.32 \mu \mathrm{m}$, with a maximum absorptivity of 0.98 , which indicates that the absorption capacity of the flat plate increases with wavelength and then decreases sharply. It is consistent with the trend that the imaginary part of the complex permittivity of $\mathrm{Ag}$ first decreases and then increases and reaches a minimum at $0.32 \mu \mathrm{m}$, as shown in Figure 2. Since the two curves are almost the same, the FEM calculation result is consistent with the analytical solution. Therefore, the following calculations are all carried out using the finite element method.

\subsection{Computational Domain of the Periodic Cubic Structure}

As shown in Figure 4, the computational domain of the periodic cubic is taken to be unit cubic connected structure with unit cubic cell length $a$ and an internal cubic pore cell length $b$. Therefore, the effective cell size can be calculated by $d_{\mathrm{p}}=\left[\left(18 a b^{2}-12 b^{3}\right) / \pi\right]^{1 / 3}$ and the porosity can be obtained by $\varphi=\left(3 a b^{2}-2 b^{3}\right) / a^{3}$. The incident surface to the top of the cubic is set at a distance $h=250 \mathrm{~nm}$, with periodic boundary conditions on all surfaces except the top and bottom, respectively, which are set as the emitting and receiving ports, where the incident magnetic field strength $H=1 \mathrm{~A} / \mathrm{m}$ is given, plus a perfect absorption layer. Furthermore, the integral calculation band of thermal radiation was $2.6 \mu \mathrm{m}<\lambda<90 \mu \mathrm{m}$. The incidence angles were divided into 20,000-unit angles from 0 to $90^{\circ}$, and the frequency interval was $2 \times 10^{12} \mathrm{~Hz}$, which was used in the following calculations to ensure the calculation accuracy. 
(a)

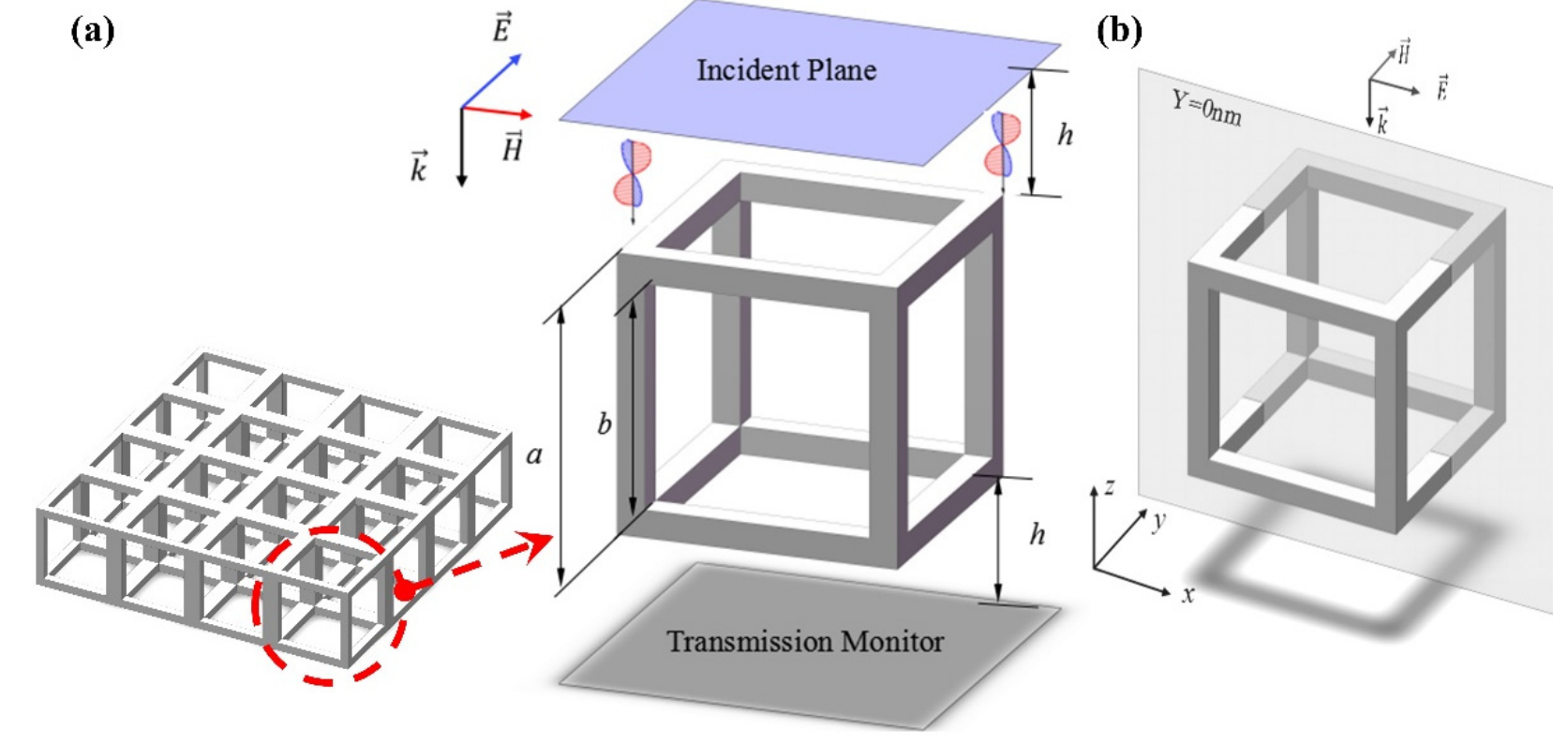

Figure 4. (a) Computational domain; (b) the research section diagram perpendicular to the direction of the magnetic field.

In order to verify the independence of the computational model, a dimensionless number $M e$ is defined, $M e=\lambda / \delta^{1 / 2}$, where $\lambda$ denotes the incident wave wavelength and $\delta$ denotes the characteristic length of elements the unit calculated domain. By adjusting the $\delta$, the absorption with wavelength for different accuracy pairs in the visible wavelength band is plotted as well as the error as shown in Figure 5. It can be obtained from Figure 5, as the value of $\mathrm{Me}$ increases, the absorbance curve keeps changing. When $\mathrm{Me} \geq 5$, the change of the absorption curve is no longer obvious with the increase of $M e$. Therefore, the smallest error is obtained when the $M e \geq 5$, which is the domain dividing into 740,000 hexahedral elements at least. As a result, the domain would be divided into around 740,000 elements in the following calculations to ensure smaller errors.

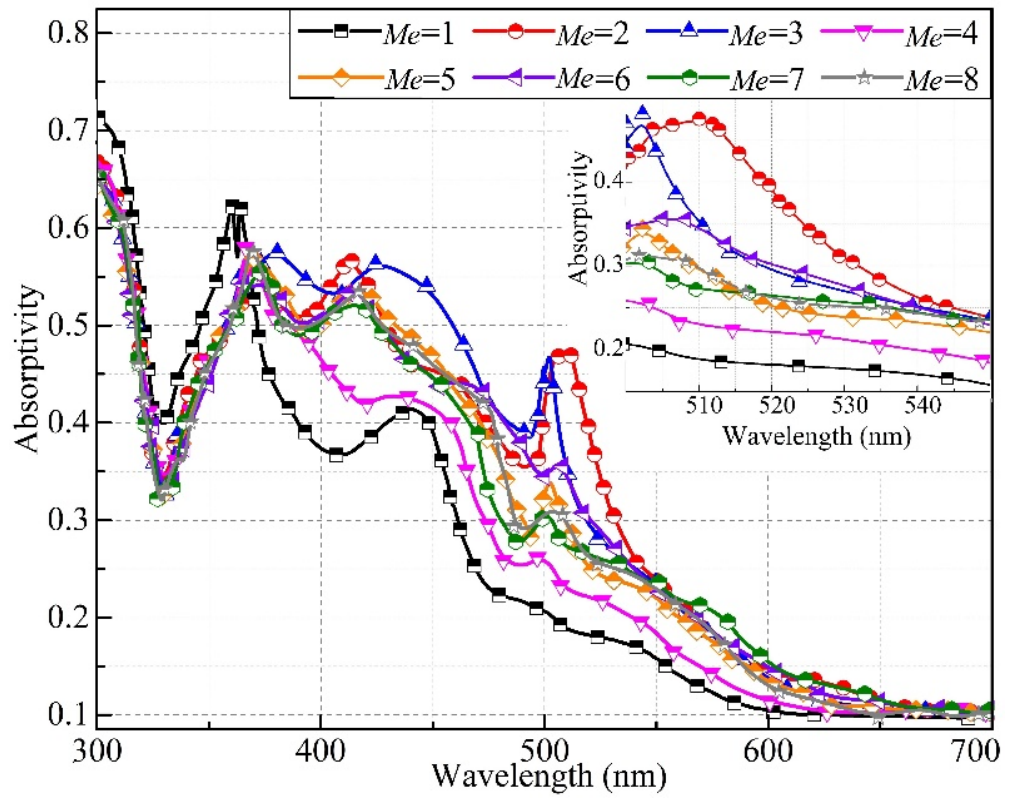

Figure 5. The Me precision analysis of computational model. 


\section{Results and Discussion}

\subsection{The Equivalent Thermal Conductivity}

In order to discuss the thermal radiation proportion in the total heat transfer process more intuitively, the equivalent thermal conductivity was chosen in this section as the evaluation index to analyze the changes of the three kinds of equivalent thermal conductivities with the cell size and porosity. Four group of higher porosities models $\varphi=0.896$, $\varphi=0.784, \varphi=0.684$ and $\varphi=0.500$ were calculated at $T=300 \mathrm{~K}$, respectively, with the temperature interval was selected as $1 \mathrm{~K}$. In addition, the integral calculation band of thermal radiation was $2.6 \mu \mathrm{m}<\lambda<90 \mu \mathrm{m}$. The thermal conductivity of pure Ag was set as $\kappa_{s}=406 \mathrm{~W} / \mathrm{m} \cdot \mathrm{K}$ followed Yong's [29] research. The spectral refractive index $n$ and spectral extinction coefficient $k$ were adopted from Yang's experimental measurements [31], as seen in Figure 2. The $\kappa_{\text {total }}, \kappa_{\text {cond }}$ and $\kappa_{\text {rad }}$ were calculated from Equations (12) and (15), respectively as a function of the size of the structure, as shown in Figure 6.
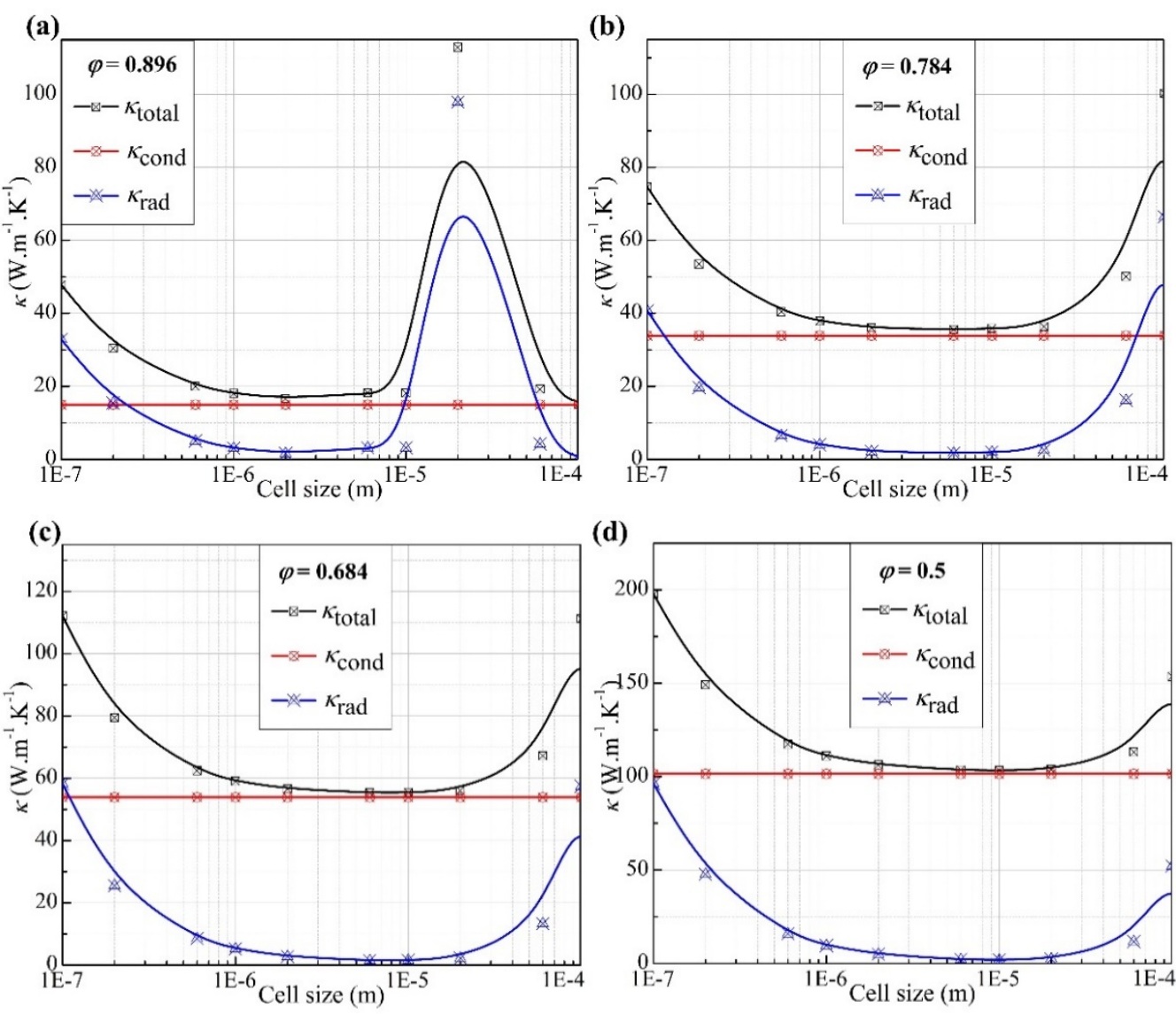

Figure 6. Equivalent thermal conductivity of high-porosity periodic cubic frame silver structure: (a) $\varphi=0.896$; (b) $\varphi=0.784 ;$ (c) $\varphi=0.684$; (d) $\varphi=0.500$.

As shown in Figure 6, the $\kappa_{\text {cond }}$ is not significantly impacted by changes in cell size $d_{\mathrm{p}}$, while the $\kappa_{\text {cond }}$ increased with the $\varphi$ decreased. This is because as the $\varphi$ decreases, the proportion of the gas phase thermal conductivity increases, which ultimately leads to a decrease in the overall $\kappa_{\text {cond }}$. For models $\varphi=0.784, \varphi=0.684$ and $\varphi=0.500$, as the structure size increases, the thermal radiation and total heat transfer decrease first and then increase, while at $\varphi=0.896$, as the structure size increases, the thermal radiation and total heat transfer decrease first and then increase to a peak before decreasing. It is because when the aperture size is comparable to the peak Planck blackbody radiation, the microscale effect is enhanced so that the total extinction coefficient decreases, resulting in the peak in Figure $6 \mathrm{a}$. When $a<1 \mu \mathrm{m}$, the larger the porosity for the same size, the larger the share of the $\kappa_{\text {total }}$ in the $\kappa_{\text {rad }}$, and the same trend of change in the $\kappa_{\text {rad }}$ and the $\kappa_{\text {total }}$. Meanwhile, 
when $\varphi=0.896, d_{\mathrm{p}}<1 \mu \mathrm{m}$, the $\kappa_{\text {rad }}$ plays a dominant role in the $\kappa_{\text {total }}$. Hence, on the basis of this set of models, the next section shall concern the reasons for this phenomenon from the perspective of the thermal radiation medium properties.

\subsection{Absorptivity, Reflectivity and Transmissivity}

In order to research the phenomenon of the large share of $\kappa_{\text {rad }}$ at micro/nano sizes, a model with cell length $a=500 \mathrm{~nm}$, an internal control cell length of $b=400 \mathrm{~nm}$ and porosity $\varphi=0.896$ is taken as an example for this paper, and the analysis of the thermal radiation properties of the medium at wavelengths comparable to the size of the structure is carried out specifically.

Firstly, the section calculates the absorptivity, reflectivity and transmissivity of infinitely large flat and periodic high porosity cubic frame structures at wavelengths in the visible and near infrared, as shown in Figure 7. For structures with finite large flat plates are shown in Figure 7a. When the wavelength is smaller than the size of the structure in the propagation direction, the reflectivity is lower and the absorption is the first to increase until it plummets close to 1 . However, when the wavelength is larger than the size of the structure in the propagation direction, the absorption decreases to close to 0 , at which point the reflectivity approaches 1 . The transmissivity is always close to 0 , and the curves of absorption and reflectivity level off when the wavelength is two times larger than the size in the propagation direction. When the wavelength approaches the size of the structure in the direction of propagation, the absorption decreases abruptly, and the reflectivity increases abruptly. This is close to the distribution curve of the real part of the dielectric constant of metallic silver, and in the infrared band the dielectric constant of silver is greater than 1 , close to total reflection, which is consistent with a reflectivity close to 1 in the infrared band.

(a)

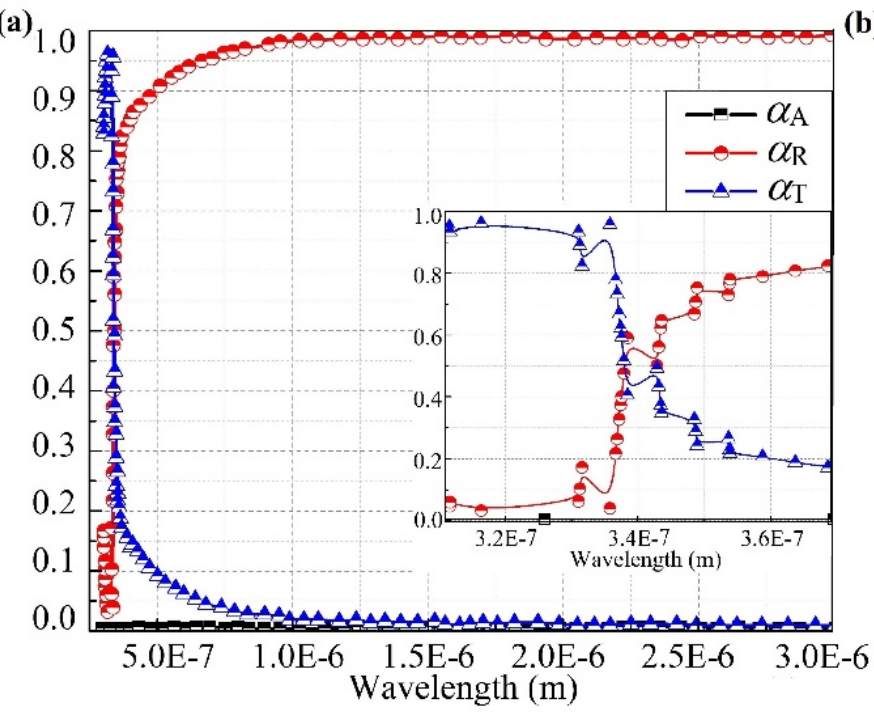

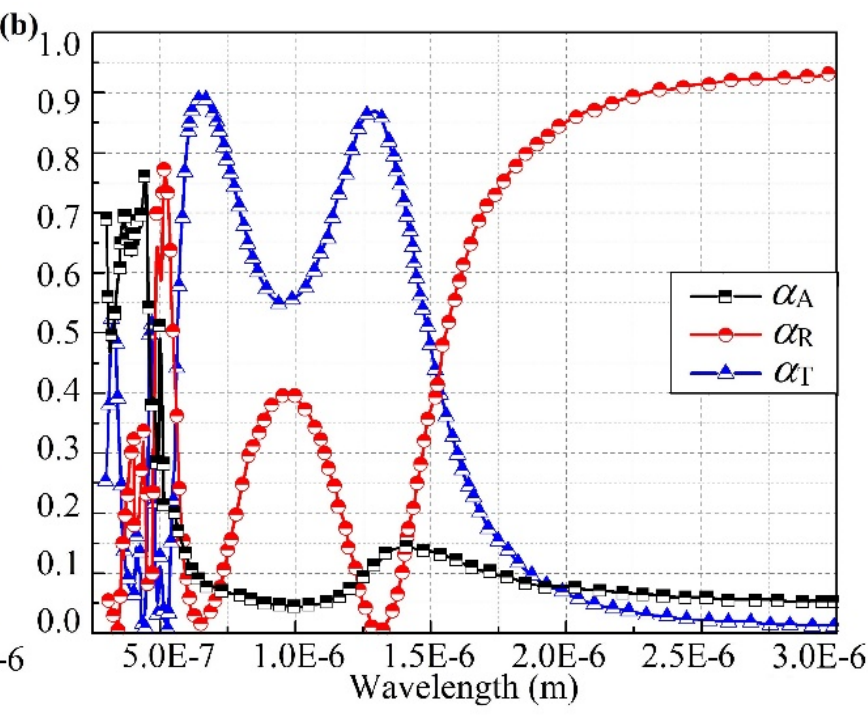

Figure 7. (a) The absorptance, reflectivity and transmissivity of Infinite plate; (b) The absorptance, reflectivity and transmissivity of periodic cubic frame silver.

As shown in Figure $7 \mathrm{~b}$, the reflection, absorption and transmission rates of the periodic high porosity cubic frame structure in the visible and mid-infrared wavelengths differ significantly from those of an infinite flat plate of the same thickness. At visible wavelengths, the oscillation of the periodic cubic frame structure with a structure size of $500 \mathrm{~nm}$ is more severe, while its absorption, reflection and transmission rates do not show monotonicity in their variation with wavelength. At wavelengths greater than $2 \mu \mathrm{m}$, the absorption, reflection and transmission rates of this structure are close to those of the flat plate structure. However, the absorption of the structure increases first and then decreases in the near infrared band, with a maximum value of 0.178 at $1.425 \mu \mathrm{m}$ wavelength, after which it 
decreases to a level close to 0.05 . Although this absorption is not high, it is a significant modulation of the absorption compared to the zero absorption of the flat structure in this band. Meanwhile, the peaks and troughs of the reflectivity and transmissivity of the structure in the NIR band correspond to the same wavelength. The reflectivity has two troughs at $0.7 \mu \mathrm{m}$ and $1.28 \mu \mathrm{m}$, where the reflectivity is nearly 0 . The transmissivity has two peaks, which correspond to a transmissivity of nearly 0.9 , respectively. The reflectivity peaks coincide with the transmissivity peaks, where the reflectivity reaches a maximum of 0.4 at a wavelength of $0.96 \mu \mathrm{m}$, and the transmissivity is at a minimum of 0.525 . In order to better investigate the NIR band modulation properties of periodic high porosity microstructures, the following is a mechanistically sound explanation of the spectral radiation properties at the reflectivity and absorptivity peaks in this band, respectively.

\subsection{Spectral Radiation Properties at the Reflectivity Peak}

In order to clearly illustrate the electric field direction and magnetic field strength, at different locations of cross sections within the calculated cell at the reflectivity and absorptivity peaks, cross sections are now taken at $Y=0 \mathrm{~nm}, \mathrm{Y}=100 \mathrm{~nm}, \mathrm{Y}=200 \mathrm{~nm}$, $\mathrm{Y}=225 \mathrm{~nm}$ and $\mathrm{Y}=250 \mathrm{~nm}$, respectively, as shown in Figure 4, with the centre of the cell as the origin, along the vertical one magnetic field direction. Where the black wire frame indicates the plane obtained by targeting the plane to the material, that is the area within the frame indicates the magnetic and electric field distribution within the material.

It can be seen from Figure 8, the distribution of the electric field direction and magnetic field intensity for each cross-section at the peak of absorptivity, which is at a wavelength of $0.96 \mu \mathrm{m}$. Where the direction of electric field transmission inside the cubic frame structure changes at the cross-section $Y=0 \mathrm{~nm}$ and $Y=100 \mathrm{~nm}$ cross sections. Due to the coupling of the induced magnetic field formed by induction with the incident magnetic field an inverse magnetic resonance is formed, this resonance is a change in the direction of the electric field transmission inside. Due to the small size of the structure a closed circuit cannot be formed, but the induced magnetic field formed by it weakens the magnetic field there; thus the magnetic field strength at the four cubic sections is lower. The phenomenon is most pronounced in the section above the structure. At the $\mathrm{Y}=200 \mathrm{~nm}$ cross-section, this plane is just above the surface of the cubic frame structure. As shown in Figure 8c, the direction of the electric field near the surface of the prism is deflected and the resonance can be observed at the surface of the prismatic structure. It decays rapidly in the interior, which is typical Surface Plasmon Polariton (SPP). The SPP resonance is generated by the incident magnetic field coupled to a locally induced electric field and shows an exponential decay of the surface swift electromagnetic waves propagating along the interface between the free space and the structure and forming a resonance with the plasma excitations on the surface of the silver cubic frame structure. In comparison with Figure $8 \mathrm{c}-\mathrm{e}$, it can be observed that the SPP resonance is more pronounced the closer the central section of the cubic cell.

\subsection{Spectral Radiation Properties at the Absorptivity Peak}

In order to study the peak absorptivity of the cubic frame structure, cross sections were taken at positions $Y=0 \mathrm{~nm}, \mathrm{Y}=100 \mathrm{~nm}, \mathrm{Y}=200 \mathrm{~nm}, \mathrm{Y}=225 \mathrm{~nm}$ and $\mathrm{Y}=250 \mathrm{~nm}$ at a wavelength of $1.425 \mu \mathrm{m}$ as shown in Figure 3, respectively, and the logarithmic distribution of the electric field direction and magnetic field intensity of each cross section was investigated, and cloud plots were made.

As shown in Figure 9, the distribution of the electromagnetic field at each cross-section is broadly similar to Figure 8, where the surface plasma resonance effect triggers an enhancement and weakening of the induced magnetic field, with a significant attenuation at the centre along the propagation direction compared to Figure 8 . When the electromagnetic wave is incident on the surface of the periodic cubic frame, it is known from the law of flutters that a time-varying electromagnetic wave will form an induced current inside the cavity. This current subsequently generates an induced magnetic field in the opposite di- 
rection, which couples with the incident magnetic field to form an anti-magnetic resonance. This resonance is known as the and Magnetic Polaritons (MPs) resonance, which forms a parallel electric field direction current inside the internal cavity. Meanwhile, comparing the absorption and reflection peaks in Figure 7, it can be seen that the absorption peak at a wavelength of $1.425 \mu \mathrm{m}$ is wider, which is typical of the magnetic polarization excitation phenomenon. Moreover, the excitation phenomenon does not change with the angle of incidence, which is of great importance in industrial production.

(a)

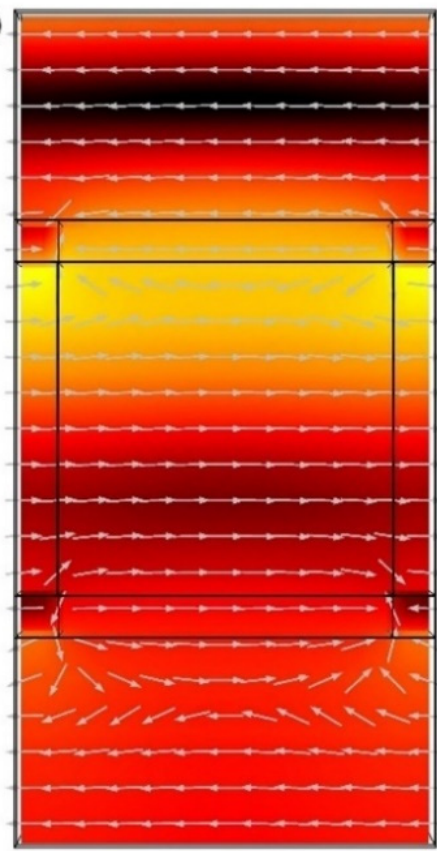

(b)

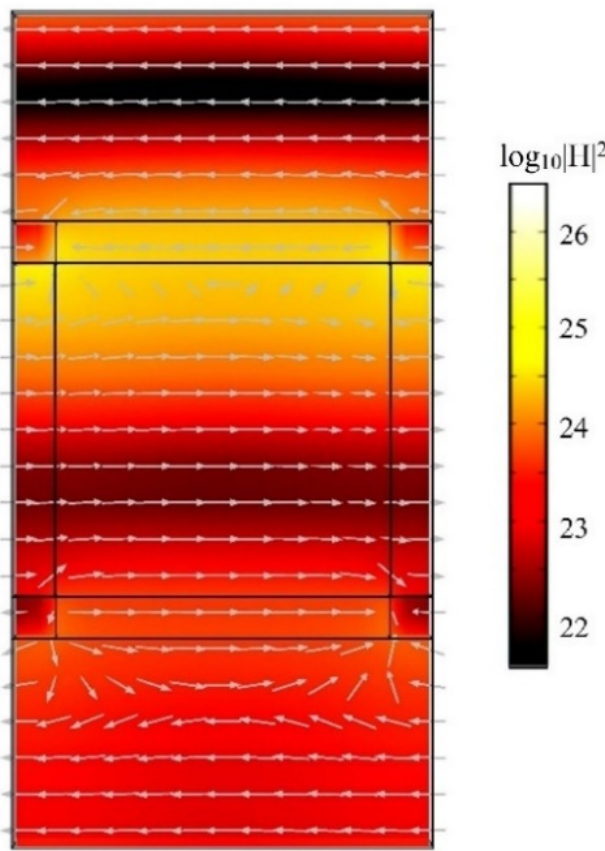

(c)

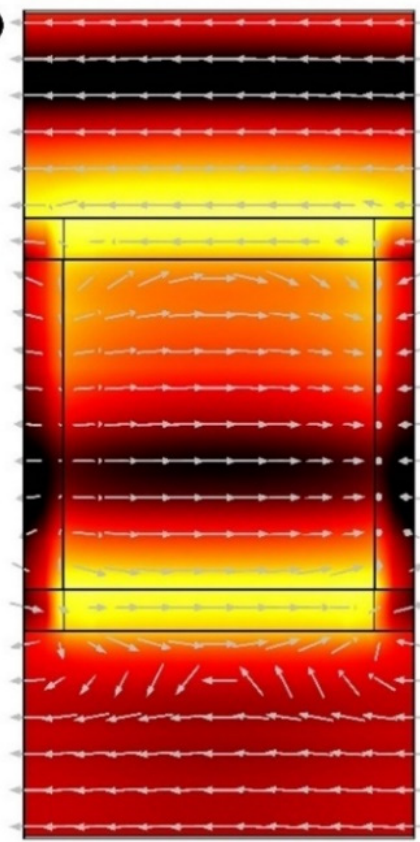

(d)

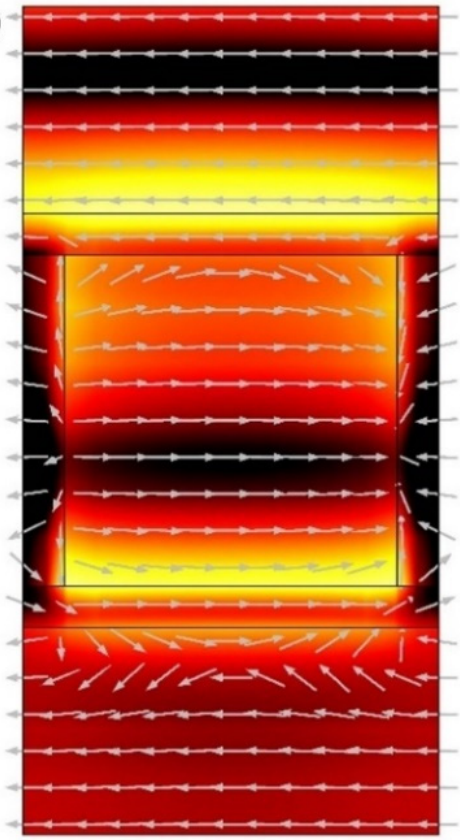

(e)

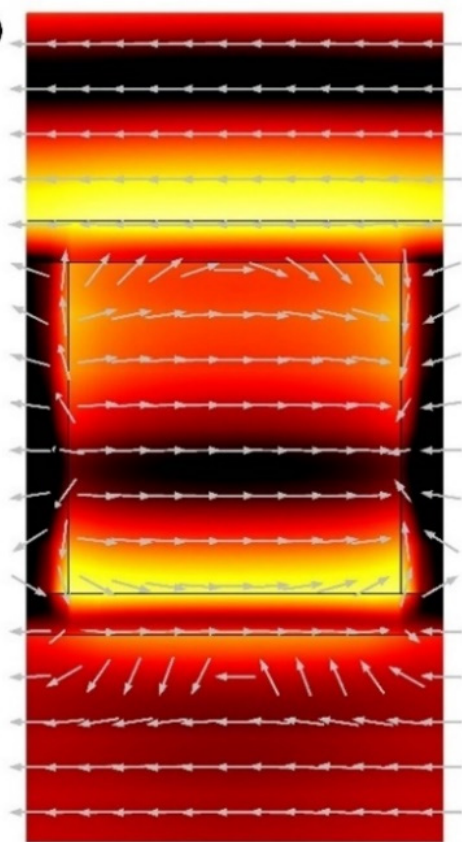

Figure 8. Magnetic and electric fields intensity distribution at the different compute region at the wavelength of $0.96 \mu \mathrm{m}$ : (a) $Y=0 \mathrm{~nm}$, (b) $Y=100 \mathrm{~nm}$, (c) $Y=200 \mathrm{~nm}$, (d) $Y=225 \mathrm{~nm}$, (e) $Y=250 \mathrm{~nm}$. 
(a)

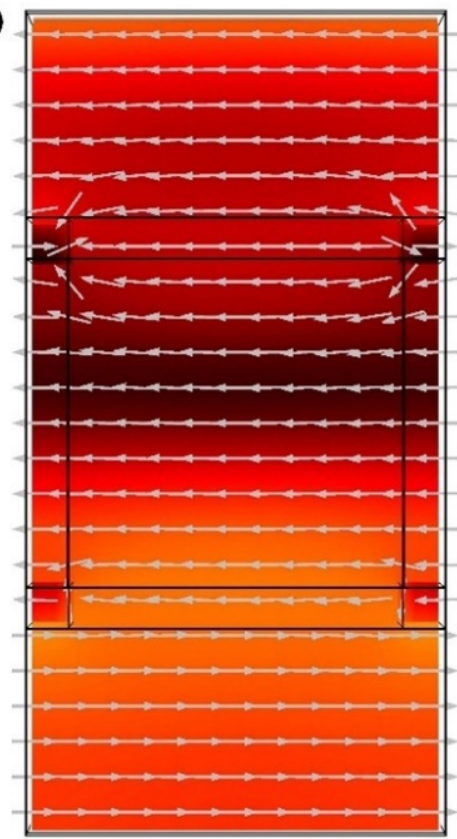

(b)

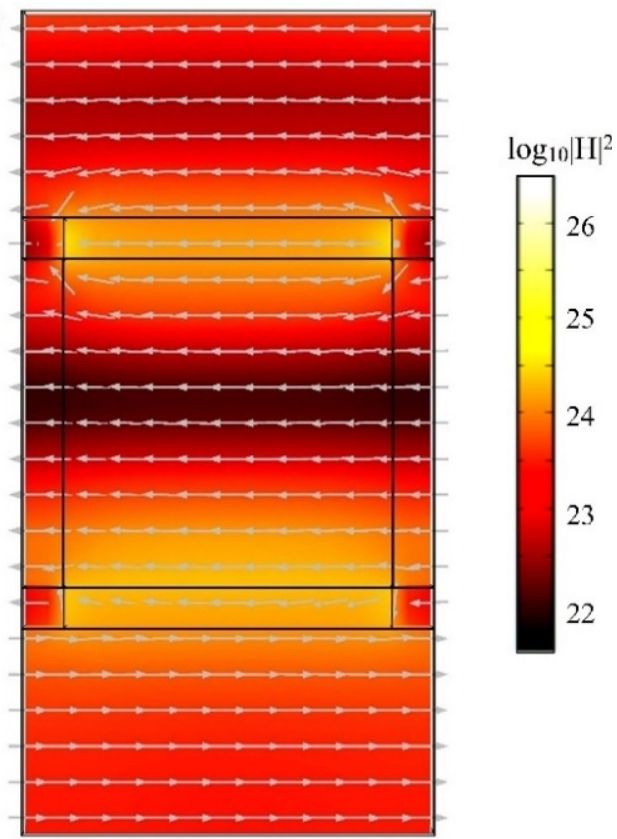

(c)

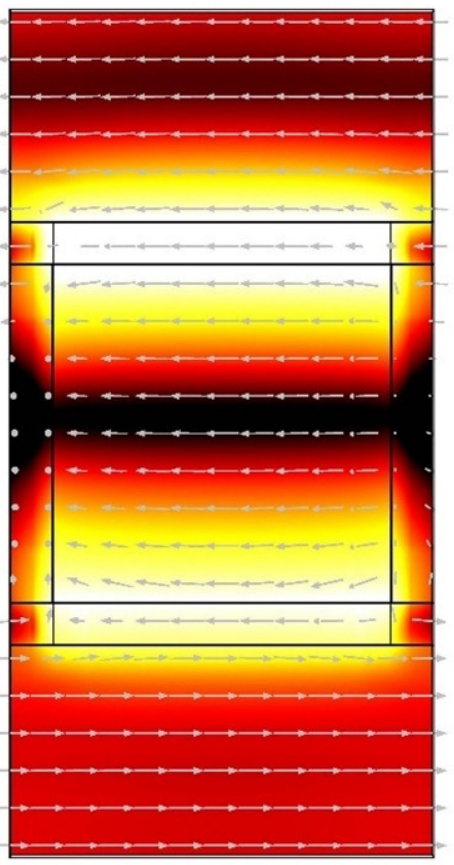

(d)

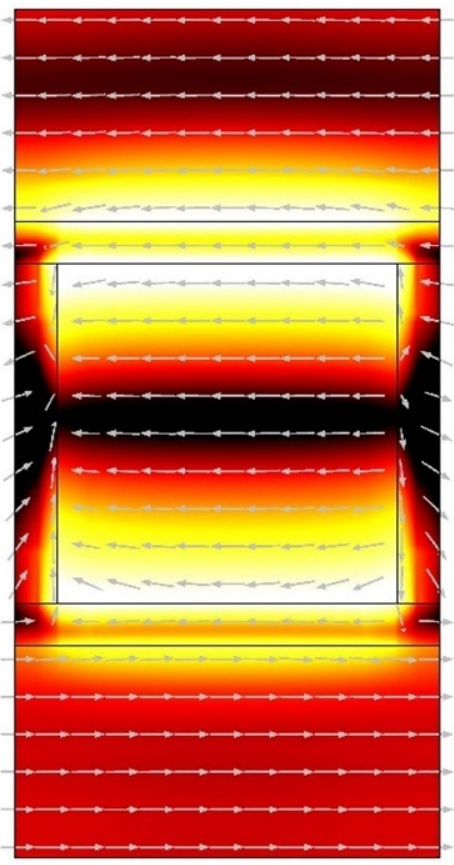

(e)

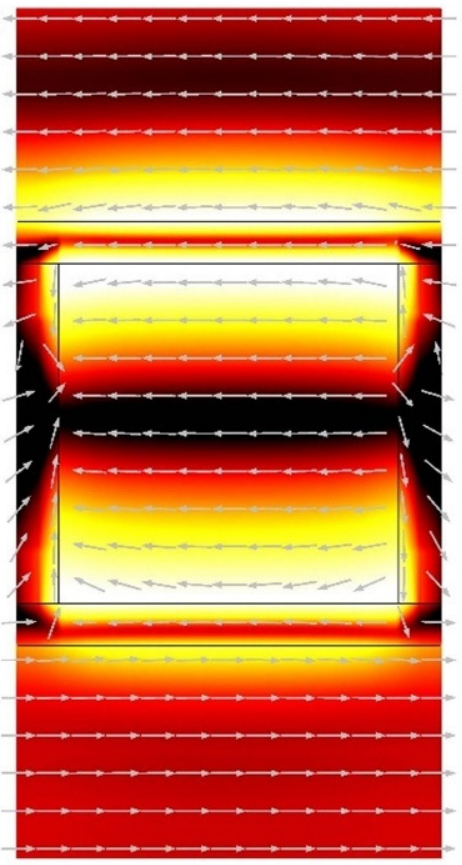

Figure 9. Magnetic and electric fields intensity distribution at the different compute region at the wavelength of $1.425 \mu \mathrm{m}$ : (a) $\mathrm{Y}=0 \mathrm{~nm}$, (b) $\mathrm{Y}=100 \mathrm{~nm}$, (c) $\mathrm{Y}=200 \mathrm{~nm}$, (d) $\mathrm{Y}=225 \mathrm{~nm}$, (e) $\mathrm{Y}=250 \mathrm{~nm}$.

\section{Conclusions}

This paper has calculated the equivalent thermal conductivity of periodic cubic frame silver with porosities of $0.896,0.784,0.684$ and 0.500 , respectively and cell size from $100 \mathrm{~nm}$ to $100 \mu \mathrm{m}$ using the finite element method. In order to explain the effect of microscale effects on the equivalent radiative thermal conductivity, this work further investigated the reflectivity, absorptivity and transmissivity of periodic cubic frame silver at the incident band of $300 \mathrm{~nm}-3 \mu \mathrm{m}$. Then, the magnetic field strength and electric field direction were analyzed at different cross sections in the band at the peak of absorptivity and reflectivity, in order to explaining the thermal radiation transmission mechanism. The conclusions are consulted as follows: 
1. In the equivalent thermal conductivity of a periodic cubic frame structure, the smaller the cell size of the structure, the larger the proportion of thermal radiation. When the structure size is comparable to the characteristic wavelength, the equivalent radiative thermal conductivity increases sharply.

2. Periodic cubic frame sliver has better thermal radiation properties in the near infrared band while fluctuates a lot in the visible band.

3. The reflection of the periodic cubic frame sliver enhanced at $680 \mathrm{~nm}-1.28 \mu \mathrm{m}$, with the strongest reflectivity happened at $960 \mathrm{~nm}$ wavelength; and the absorption enhanced at a wavelength band of $1 \mu \mathrm{m}-1.7 \mu \mathrm{m}$, as the strongest absorption happened when wavelength is $1.425 \mu \mathrm{m}$.

4. The thermal radiation mechanism of periodic cubic frame structure was explained by the SPP and MPs resonance jointly form a resonance in the near infrared band, while the cavity formed inside the MPs excitation of the absorption peak phenomenon.

Author Contributions: Conceptualization, H.Z. and H.Y.; methodology, H.Y.; investigation, H.W.; data curation, D.Z.; writing—original draft preparation, H.Y.; writing—review and editing, D.Z. and H.W.; funding acquisition, H.Z. All authors have read and agreed to the published version of the manuscript.

Funding: This research was funded by National Natural Science Foundation of China, grant number 51776050, the National Key R\&D Program of China, grant number 2020YFB1901900, and the China Scholarship Council, grant number 201806120171.

Institutional Review Board Statement: Not applicable.

Informed Consent Statement: Not applicable.

Data Availability Statement: The data that support the findings of this study are available from the corresponding author upon reasonable request.

Conflicts of Interest: The authors declare no conflict of interest.

\section{Nomenclature}

$\begin{array}{lll}A & \text { unit cross-sectional area } & \mathrm{m}^{2} \\ A^{\prime} & \text { area } & \mathrm{m}^{2} \\ a & \text { unit cubic cell length } & \mathrm{m} \\ B & \text { magnetic flux density } & \mathrm{Wb} / \mathrm{m}^{2} \\ b & \text { internal cubic pore length } & \mathrm{m} \\ c & \text { specific heat capacity } & (\mathrm{J} \cdot \mathrm{kg}) / \mathrm{K} \\ d & \text { thickness } & \mathrm{m} \\ D & \text { electric displacement vector } & \mathrm{C} / \mathrm{m}^{2} \\ E & \text { electric field vector } & \mathrm{V} / \mathrm{m} \\ H & \text { magnetic field vector } & \mathrm{A} / \mathrm{m} \\ h & \text { incident distance } & \mathrm{m} \\ J & \text { current density } & \mathrm{A} / \mathrm{m}^{2} \\ k & \text { spectral extinction coefficient } & - \\ k_{\mathrm{B}} & \text { Boltzmann constant } & \mathrm{J} / \mathrm{K} \\ M e & \text { dimensionless number } & - \\ n & \text { refractive index } & - \\ n & \text { heat flow vector } & \\ N & \text { particle numbers } & - \\ r & \text { reflection coefficient } & \\ R & \text { internal pore radius } & \mathrm{m} \\ S & \text { incident energy flow } & \mathrm{W} \\ T & \text { temperature } & \mathrm{K} \\ t & \text { time } & \mathrm{s} \\ t & \text { transmission coefficient } & \mathrm{m} 2 \\ Q & \text { cross-section } & \end{array}$




\begin{tabular}{|c|c|c|}
\hline$q$ & density of heat source & $\mathrm{W} / \mathrm{m}^{2}$ \\
\hline$V$ & volume & $\mathrm{m}^{3}$ \\
\hline$W$ & rate & - \\
\hline$x, y, z$ & coordinate direction & - \\
\hline$\alpha$ & coefficient & - \\
\hline$\beta$ & coefficient & - \\
\hline$\delta$ & grid size & $\mathrm{m}$ \\
\hline$\rho$ & volume charge density/density & $\mathrm{C} / \mathrm{m}^{3} \mathrm{~kg} / \mathrm{m}^{3}$ \\
\hline$\kappa$ & thermal conductivity & $\mathrm{W} /(\mathrm{m} \cdot \mathrm{K})$ \\
\hline$\varepsilon$ & permittivity & $\mathrm{F} / \mathrm{m}$ \\
\hline$\hbar$ & Planck constant divided by $2 \pi$ & $\mathrm{J} \cdot \mathrm{s}$ \\
\hline$\sigma_{\mathrm{SB}}$ & Steven-Boltzmann constant & - \\
\hline$\lambda$ & wavelength & $\mathrm{m}$ \\
\hline$\varphi$ & porosity & - \\
\hline$\mu$ & permeability & $\mathrm{H} / \mathrm{m}$ \\
\hline \multicolumn{3}{|c|}{ Subscript } \\
\hline A & absorption & \\
\hline $\mathrm{a}$ & absorption & \\
\hline cond & conduction & \\
\hline $\mathrm{e}$ & extinction & \\
\hline $\mathrm{p}$ & Pore/ cell size & \\
\hline $\mathrm{R}$ & reflection & \\
\hline $\mathrm{r}$ & radius direction & \\
\hline $\mathrm{rad}$ & thermal radiation & \\
\hline $\mathrm{s}$ & scattering & \\
\hline $\mathrm{T}$ & transmission & \\
\hline total & total heat transfer & \\
\hline $\mathrm{V}$ & volume & \\
\hline $\mathrm{w}$ & equivalent wall size & \\
\hline \multicolumn{3}{|c|}{ Superscript } \\
\hline * & complex vector & \\
\hline a & absorption & \\
\hline $\mathrm{i}$ & incident & \\
\hline $\mathrm{s}$ & scattering & \\
\hline
\end{tabular}

\section{References}

1. Tien, C.L. Microscale Energy Transfer; CRC Press: Boca Raton, FL, USA, 1997.

2. Cravalho, E.G.; Tien, C.L.; Caren, R.P. Effect of small spacings on radiative transfer between two dielectrics. J. Heat Transf. 1967, 89, 351-358. [CrossRef]

3. Mulet, J.P.; Joulain, K. Enhanced radiative heat transfer at nanometric distances. Micro. Therm. Eng. 2002, 6, 209-222. [CrossRef]

4. Bikbaev, R.G.; Vetrov, S.Y.; Timofeev, I.V. Hybrid Tamm and surface plasmon polaritons in resonant photonic structure. J. Quant. Spectrosc. Radiat. Transf. 2020, 253, 107156. [CrossRef]

5. Nakkach, M.; Duval, A.; Ea-Kim, B.; Moreau, J.; Canva, M. Angulo-spectral surface plasmon resonance imaging of nanofabricated grating surfaces. Opt. Lett. 2010, 35, 2209-2211. [CrossRef]

6. Ge, C.X.; Wu, Z.S.; Bai, J.; Gong, L. Effect of nanoscale roughness on optical trapping properties of surface plasmon polaritons exerted on nanoparticle. J. Quant. Spectrosc. Radiat. Transf. 2018, 219, 339-349. [CrossRef]

7. Hafeli, A.K.; Rephaeli, E.; Fan, S.; Cahill, D.G.; Tiwald, T.E. Temperature dependence of surface phonon polaritons from a quartz grating. J. Appl. Phys. 2011, 110, 043517. [CrossRef]

8. Van Zwol, P.J.; Joulain, K.; Ben-Abdallah, P.; Chevrie, J. Phonon polaritons enhance near-field thermal transfer across the phase transition of $\mathrm{VO}_{2}$. Phys. Rev. B 2011, 84, 161413. [CrossRef]

9. Painter, O.; Lee, R.K.; Scherer, A.; Yariv, A.; O’Brien, J.D.; Dapkus, P.D.; Kim, I. “Two-dimensional photonic band-gap defect mode laser. Science 1999, 284, 1819-1821. [CrossRef] [PubMed]

10. Chen, Y.Y.; Liao, H.Y.; Shi, J.L.; Wang, Q. Unusual photon tunneling in the frustrated total internal reflection structure including indefinite metamaterials. J. Opt. 2010, 12, 292-295. [CrossRef]

11. Lee, B.J.; Wang, L.P.; Zhang, Z.M. Coherent thermal emission by excitation of magnetic polaritons between periodic strips and a metallic film. Opt. Express. 2008, 16, 11328-11336. [CrossRef]

12. Lee, B.J.; Zhang, Z.M. Coherent thermal emission from modified periodic multilayer structures. J. Heat Transf. 2007, 129, 17-26. [CrossRef] 
13. Wang, A.H.; Hsu, P.F.; Chen, Y.B.; Cai, J.J. Effects of nanoscale features on infrared radiative properties of heavily doped silicon complex gratings. Sci. China Technol. Sci. 2010, 53, 2207-2214. [CrossRef]

14. Qiu, J. Infrared Radiative Properties of Micro Scale Structures of Near-Surface. Ph.D. Dissertation, Harbin Institute of Technology, Harbin, China, 2012.

15. Huang, J.G. Research on the Control Methods of Infrared Thermal Radiation Characteristics of Micostructured Surface. Ph.D. Dissertation, Nanjing University of Science and Technology, Nanjing, China, 2015.

16. Huang, J.G.; Xuan, Y.M.; Li, Q. Narrow-band spectral features of structured silver surface with rectangular resonant cavities. J. Quant. Spectrosc. Radiat. Transf. 2011, 112, 839-846. [CrossRef]

17. Liu, X.; Xia, H.; Xuan, Y. Effects of near-field photon tunneling on the performance of photon-enhanced thermionic emission energy conversion. J. Quant. Spectrosc. Radiat. Transf. 2019, 222, 223-228. [CrossRef]

18. Zheng, Z.; Wang, A.; Xuan, Y. Spectral tuning of near-field radiative heat transfer by graphene-covered metasurfaces. J. Quant. Spectrosc. Radiat. Transf. 2018, 208, 86-95. [CrossRef]

19. Liu, B.; Xia, X.L.; Sun, C.; Chen, X. Analysis on spectral radiative properties of micro-scaled rough ligament surface inside open-cell nickel foam. Int. J. Heat Mass Transf. 2019, 145, 118773. [CrossRef]

20. Liu, B.; Xia, X.L.; Zhang, X.; Sun, C. Spectral radiative properties of skeleton inner structure of ceramic foam based on ordered opal structure model. J. Quant. Spectrosc. Radiat. Transf. 2019, 224, 279-288. [CrossRef]

21. Wang, C.H.; Yi, H.L.; Tan, H.P. Discontinuous finite element method for vector radiative transfer. J. Quant Spectrosc. Radiat. Transf. 2017, 189, 383-397. [CrossRef]

22. Yu, H.; Zhang, H.; Dai, Z.; Xia, X. Design and analysis of low emissivity radiative cooling multilayer films based on effective medium theory. ES Energy Environ. 2019, 6, 69-77. [CrossRef]

23. Wang, C.H.; Qu, L.; Zhang, Y.; Yi, H.L. Three-dimensional polarized radiative transfer simulation using discontinuous finite element method. J. Quant. Spectrosc. Radiat. Transf. 2018, 208, 108-124. [CrossRef]

24. Zhang, Z.M. Nano/Microscale Heat Transfer; McGraw-Hill: New York, NY, USA, 2007.

25. Bohren, C.F.; Huffman, D.R. Absorption and Scattering of Light by Small Particles; John Wiley \& Sons: Weinheim, Germany, 2008.

26. Rui, X.; Wang, G.; Zhang, J. Transfer Matrix Method for Multibody Systems: Theory and Applications; John Wiley \& Sons: Hoboken, NJ, USA, 2018.

27. Yu, H.; Zhang, H.; Xia, X. A fractal-skeleton model of high porosity macroporous aluminum and its heat transfer characterizes. J. Therm. Anal. Calorim. 2020, 1, 1-10. [CrossRef]

28. Yu, H.; Zhang, H.; Buahom, P.; Liu, J.; Xia, X.; Park, C.B. Prediction of thermal conductivity of micro/nano porous dielectric materials: Theoretical model and impact factors. Energy 2021, 121140. [CrossRef]

29. Huang, H.C.; Usmani, A.S. Finite Element Analysis for Heat Transfer: Theory and Software; Springer Science \& Business Media: Berlin, Germany, 2012.

30. Young, D.H. University Physics, 7th ed.; Addison Wesley: Boston, MA, USA, 1992.

31. Yang, H.U.; D'Archangel, J.; Sundheimer, M.L.; Tucker, E.; Boreman, G.D.; Raschke, M.B. Optical dielectric function of silver. Phys. Rev. B 2015, 91, 235137. [CrossRef] 